\title{
Topological, noninertial and spin effects on the 2D Dirac oscillator in the presence of the Aharonov-Casher effect
}

\author{
R. R. S. Oliveira ${ }^{a}$ \\ Departamento de Física, Universidade Federal do Ceará, Campus do Pici, Fortaleza, CE 60455-760, Brazil
}

Received: 1 July 2019 / Accepted: 20 August 2019 / Published online: 29 August 2019

(C) The Author(s) 2019

\begin{abstract}
In the present paper, we investigate the influence of topological, noninertial and spin effects on the 2D Dirac oscillator in the presence of the Aharonov-Casher effect. Next, we determine the two-component Dirac spinor and the relativistic energy spectrum for the bound states. We observe that this spinor is written in terms of the confluent hypergeometric functions and this spectrum explicitly depends on the quantum numbers $n$ and $m_{l}$, parameters $s$ and $\eta$ associated to the topological and spin effects, quantum phase $\Phi_{A C}$, and of the angular velocity $\Omega$ associated to the noninertial effects of a rotating frame. In the nonrelativistic limit, we obtain the quantum harmonic oscillator with two types of couplings: the spin-orbit coupling and the spin-rotation coupling. We note that the relativistic and nonrelativistic spectra grow in absolute values as functions of $\eta, \Omega$, and $\Phi_{A C}$ and its periodicities are broken due to the rotating frame. Finally, we compared our problem with other works, where we verified that our results generalizes some particular planar cases of the literature.
\end{abstract}

\section{Introduction}

Since that the relativistic version of the quantum harmonic oscillator (QHO) for spin-1/2 particles was formulated in the literature in 1989 by M. Moshinsky and A. Szczepaniak, the so-called Dirac oscillator (DO) [1], several works on this model have been and continue being performed in different areas of physics, such as in thermodynamics [2,3], nuclear physics [4-6], quantum chromodynamics [7,8], quantum optics $[9,10]$, and graphene physics [11-13]. Besides that, the OD also is studied in other interesting physical contexts, such as in quantum phase transitions $[14,15]$, noncommutative spaces [16,17], minimal length scenario [18,19], supersymmetry [20,21], etc. To model the DO, it is necessary inserted into free Dirac equation (DE) the following nonmin-

\footnotetext{
a e-mail: rubensfq1900@gmail.com
}

imal coupling $\mathbf{p} \rightarrow \mathbf{p}-i m_{0} \omega \beta \mathbf{r}$, where $m_{0}$ is the rest mass of the oscillator with angular frequency $\omega>0$ and $\mathbf{r}$ is the position vector [1]. In 2013, the DO was verified experimentally by J. A. Franco-Villafañe et al [22]. Recently, the DO was studied in the context of the position-dependent mass [23], in the presence of the Aharonov-Bohm-Coulomb system [24], and of topological defects $[25,26]$.

On the other hand, the study of noninertial effects due to rotating frames have been widely investigated in the literature since to 1910 decade [27], where the best-known effects are the Sagnac [28], Barnett [29], Einstein-de Hass [30] and Mashhoon [31] effects. In the last years, nonineral effects have also been investigated in some condensed matter systems, such as in the quantum Hall effect [32,33], BoseEinstein condensates [34,35], fullerene molecules [36,37], and in atomic gases $[38,39]$. In addition, the study of noninertial effects in relativistic quantum systems also gained relevance and focus of investigations in recent years [4044]. In particular, the DE in a rotating frame has several interesting applications, for instance, is applied in physical problems involving spin currents [45,46], Sagnac and Hall effects [47,48], chiral symmetry [49], external magnetic fields [50,51], fullerene molecules [52-54], nanotubes and carbon nanocons $[55,56]$, and so on.

In literature, the first formal approach on nonrelativistic neutral quantum particles with magnetic dipole moment (MDM) interacting with external electric fields was made by Y. Aharonov and A. Casher [57], where was verified theoretically that the wave function of the neutral particle acquires a topological quantum phase due to interaction with the field, even the force of Lorentz being null. This peculiar quantum effect is known currently as Aharonov-Casher (AC) effect [58,59]. Next, some researchers formulated the relativistic AC effect via DE [60-62]. Moreover, the DE for spin-1/2 particles in the presence of external electric fields is study in connection with the graphene $[63,64]$, fermions pair production $[65,66]$, noncommutative quantum electrodynamics 
[67], vacuum instability [68], magnetic fields in conical and flat spacetimes [69-71], electric dipole moment [72,73], and so on. It is important to mention that the DO was originated through of neutral Dirac particles with MDM interacting with external electric fields [74].

The present paper has as its goal to study the influence of topological, noninertial and spin effects on the relativistic and nonrelativistic quantum dynamics of the 2D DO in the presence of the AC effect. To include the topological and noninertial effects in our problem, we rotate our system in the form $\varphi \rightarrow \varphi+\Omega t$, where $\Omega$ is the constant angular velocity of the rotating frame, and we use the cosmic string background (gravitational topological defect) described by a deficit angle $\eta$, where $\eta=1-4 M$ and $M$ is the linear mass density of the cosmic string [75-77]. Now, to include the spin effects, we modify a of the Dirac matrices in the form $\gamma_{2} \rightarrow s \gamma_{2}$, where the parameter $s$ (spin parameter) characterizes the two spin states of the DO, with $s=+1$ for spin "up" and $s=-1$ for spin "down", respectively $[78,79]$. Besides that, it is important to report that the first papers that studied the DO interacting with a topological defect (cosmic string) in the presence of the Aharonov-Bohm and AC effects are found in Refs. [80,81]. Last but not least, the topological and noninertial effects analogous to the of this paper were recently applied in nonrelativistic quantum dots [82].

This work is organized as follows. In Sect. 2, we introduce the cosmic string background and the configuration of the electric field in the rotating frame. In Sect. 3, we investigate the influence of topological, noninertial and spin effects on the relativistic quantum dynamics of the 2D DO in the presence of the AC effect. Next, we determine the Dirac spinor and the relativistic energy spectrum for the bound states of the system. In Sect. 4, we analyze the nonrelativistic limit of our results. Finally, in Sect. 5 we present our conclusions. In this work, we use the natural units where $\hbar=c=G=1$ and the spacetime with signature $(+---)$.

\section{The rotating electric cosmic string background}

In this section, we configure a curved spacetime in a rotating frame, whose spacetime chosen is the cosmic string spacetime. In cylindrical coordinates, the line element for this topological object is written as [75-77]

$d s^{2}=d T^{2}-d R^{2}-\eta^{2} R^{2} d \Phi^{2}-d Z^{2}$,

where the deficit angle $\eta$ is defined in the range $0<\eta<1$. In particular, the geometry characterized by the line element (1) has a conical singularity that gives rise to a curvature centered on the cosmic string axis ( $Z$-axis), however, in all other places the curvature is null $[75,76]$. This conical singularity is represented by the following curvature tensor
$R_{\rho, \varphi}^{\rho, \varphi}=\frac{1-\eta}{4 \eta} \delta_{2}(\mathbf{r})$

where $\delta_{2}(\mathbf{r})$ is the Dirac delta. For instance, in condensed matter physics, it is already well known that linear topological defects such as disclinations and dislocations can be described by a line element in the same that a topological defect in general relativity [83,84]. It is noteworthy that in the case of the cosmic string, the spatial part of its line element corresponds to the line element of a disclination. In this way, when we take the nonrelativistic limit of the DE, we can extend this formalism to the solid state physics context [85].

As we are interested in working in a rotating frame, we must use the following coordinate transformations

$T=t, \quad R=\rho, \quad \Phi=\varphi+\Omega t, \quad Z=z$,

where $\Omega$ is the constant angular velocity (not a angular frequency) of the rotating frame and must satisfy the condition $\Omega \rho \ll 1$. As we will see shortly, this condition will allow us to obtain exact solutions for the system. With this transformations, the line element (1) becomes [75,76,86]

$$
\begin{aligned}
d s^{2}= & \left(1-\kappa^{2}\right)\left(d t-\frac{\kappa \eta \rho}{\left(1-\kappa^{2}\right)} d \varphi\right)^{2}-d \rho^{2}-\frac{\eta^{2} \rho^{2}}{\left(1-\kappa^{2}\right)} d \varphi^{2} \\
& -d z^{2}
\end{aligned}
$$

where $\kappa \equiv \Omega \eta \rho>0$.

With the line element (4), we need to construct a local reference frame where the observers will be placed; this case, we can to define the Dirac matrices in the curved spacetime. So, a local reference frame can be built through of an noncoordinate basis given by $\hat{\theta}^{a}=e_{\mu}^{a}(x) d x^{\mu}$, which its components $e_{\mu}^{a}(x)$ satisfy the following relation $[75,76,85]$

$\mathrm{g}_{\mu \nu}(x)=e_{\mu}^{a}(x) e_{\nu}^{b}(x) \eta_{a b}, \quad(\mu, v=0,1,2,3)$,

where $g_{\mu \nu}(x)$ is the rotating curved metric tensor, $\eta_{a b}$ is the Minkowski metric tensor and the indices $a, b=0,1,2,3$ indicate the local reference frame. The components of the noncoordinate basis $e_{\mu}^{a}(x)$ are called tetrads, and whose inverse is defined as $d x^{\mu}=e_{a}^{\mu}(x) \hat{\theta}^{a}$, where $e_{\mu}^{a}(x) e_{b}^{\mu}(x)=$ $\delta^{a}{ }_{b}$ and $e_{a}^{\mu}(x) e^{a}{ }_{v}(x)=\delta_{v}^{\mu}(x)$ must be satisfied. Our interest is to build a rotating frame where there is no torque. Thus, we choose the tetrads and its inverse in the form $[75,85]$

$$
\begin{aligned}
& e_{\mu}^{a}(x)=\left(\begin{array}{cccc}
\sqrt{1-\kappa^{2}} & 0 & -\frac{\kappa \eta \rho}{\sqrt{1-\kappa^{2}}} & 0 \\
0 & 1 & 0 & 0 \\
0 & 0 & \frac{\eta \rho}{\sqrt{1-\kappa^{2}}} & 0 \\
0 & 0 & 0 & 1
\end{array}\right), \\
& e_{a}^{\mu}(x)=\left(\begin{array}{cccc}
\frac{1}{\sqrt{1-\kappa^{2}}} & 0 & \frac{\kappa}{\sqrt{1-\kappa^{2}}} & 0 \\
0 & 1 & 0 & 0 \\
0 & 0 & \frac{\sqrt{1-\kappa^{2}}}{\eta \rho} & 0 \\
0 & 0 & 0 & 1
\end{array}\right) .
\end{aligned}
$$


Note that this choice makes the 1,2 and 3 -axis of the local reference frame be parallel to the $\rho, \varphi$ and $z$-axis of the curved spacetime, respectively. With these informations about the choice of the local reference frame, we can obtain the one-form connection $\omega_{b}^{a}=\omega_{\mu b}^{a}(x) d x^{\mu}$ via MaurerCartan equations [75,85]. In the absence of the torsion (or torque), these equations are written as follows

$d \hat{\theta}^{a}+\omega^{a}{ }_{b} \wedge \hat{\theta}^{b}=0$,

where the operator $d$ is the exterior derivative and the symbol $\wedge$ means the external product. Therefore, the non-null components of the one-form connection are

$$
\begin{aligned}
& \omega_{t}{ }^{0}{ }_{1}(x)=\omega_{t}{ }^{1}{ }_{0}(x)=-\frac{\kappa \Omega \eta}{\sqrt{1-\kappa^{2}}}, \\
& \omega_{t}{ }^{1}{ }_{2}(x)=-\omega_{t}{ }^{2}{ }_{1}(x)=-\frac{\Omega \eta}{\sqrt{1-\kappa^{2}}}, \\
& \omega_{\rho}{ }^{0}{ }_{2}(x)=\omega_{\rho}{ }^{2}(x)=\frac{\Omega \eta}{\left(1-\kappa^{2}\right)}, \\
& \omega_{\varphi}{ }^{0} 1(x)=\omega_{\varphi}{ }^{1}{ }_{0}(x)=-\frac{\kappa \eta}{\sqrt{1-\kappa^{2}}}, \\
& \omega_{\varphi}{ }^{1}{ }_{2}(x)=-\omega_{\varphi}{ }^{2}{ }_{1}(x)=-\frac{\eta}{\sqrt{1-\kappa^{2}}} .
\end{aligned}
$$

Now, we focus our attention on the configuration of the electric field in the cosmic string background in a rotating frame. To introduce an electrical interaction into DE due to a neutral particle with MDM $\mu$ we must insert a nonminimal coupling into DE as follow $[81,87]$

$$
\begin{aligned}
& i \gamma^{\mu}(x) \partial_{\mu} \rightarrow i \gamma^{\mu}(x) \partial_{\mu}+\frac{\mu}{2} \sigma^{\mu \nu}(x) F_{\mu \nu}(x), \\
& \quad(\mu, v=0,1,2,3),
\end{aligned}
$$

where $\sigma^{\mu \nu}(x)=\frac{i}{2}\left[\gamma^{\mu}(x), \gamma^{\nu}(x)\right]$ is a antisymmetric tensor, being $\gamma^{\mu}(x)$ the curved gamma matrices, $F_{\mu \nu}(x)$ is the electromagnetic field tensor and $A_{\mu}(x)=e_{\mu}^{a}(x) A_{a}$ is the external electromagnetic field. Explicitly, $\sigma^{\mu \nu}(x) F_{\mu \nu}(x)$ is written in the rotating frame of the observer $(\Omega \neq 0)$ in the form [87]

$\sigma^{\mu \nu}(x) F_{\mu \nu}(x)=2 i \gamma^{0}(x) \gamma^{1}(x) E_{1}(x), \quad\left(E_{2}=E_{3}=0\right)$,

where $\gamma^{0}(x) \gamma^{1}(x)=\alpha^{1}(x)$, the electric field $E_{1}(x)$ is given by $E_{1}(x)=e_{1}^{i}(x) E_{i}(i=1,2,3)$, being $E_{i}$ the components of the electric field $\mathbf{E}=E_{i} \hat{e}_{i}$ written in the rest inertial frame of the observer ( $\Omega=0$ ), and we also assume a null magnetic field $(\mathbf{B}=0)$. Here, we consider the external electric field of the $\mathrm{AC}$ effect, in which is generated by an infinitely long wire and uniformly charged located along of the z-axis perpendicular to the polar plane. In special, this electric field is given by $\mathbf{E}=\frac{\lambda}{\eta \rho} \hat{e}_{\rho}(i=1=\rho)$, where $\lambda=\frac{\lambda_{0}}{2 \pi \epsilon_{0}}$ and $\lambda_{0}>0$ is the electric charge linear density of the wire [70,81]. Consequently, we have $E_{1}(x)=\sqrt{1-\kappa^{2}} \frac{\lambda}{\eta \rho}$, and the parameter $\eta$ arises because the wire coincides with the axis of symmetry of the cosmic string ( $z$-axis) [70,80,81].

\section{Relativistic quantum dynamics of the Dirac oscillator in the rotating electric cosmic string background}

In this section, we determine the relativistic bound-state solutions of the DO in the presence of the AC effect and under the influence of topological, noninertial and spin effects. We start our discussion initially from a system in cylindrical coordinates, and posteriorly, we turn the system in a dynamics purely planar (2D). In that way, the covariant DO in the curved spacetime for a neutral particle interacting with an external electromagnetic field is given by $[75,76,81,87]$

$$
\begin{aligned}
& {\left[i \gamma^{\mu}(x)\left(\nabla_{\mu}(x)+m_{0} \omega \rho \gamma^{0} \delta_{\mu}^{\rho}\right)+\frac{\mu}{2} \sigma^{\mu \nu}(x) F_{\mu \nu}(x)-m_{0}\right] \Psi} \\
& \quad=0
\end{aligned}
$$

where $\gamma^{\mu}(x)=e_{a}^{\mu}(x) \gamma^{a}$ and $\gamma^{a}$ are the gamma matrices defined in the inertial Minkowski spacetime, $\nabla_{\mu}(x)=\partial_{\mu}+$ $\Gamma_{\mu}(x)$ is the covariant derived, being $\Gamma_{\mu}(x)=\frac{i}{4} \omega_{\mu a b}(x) \sigma^{a b}$ the spinorial connection and the quantity $\omega_{\mu a b}(x)$ is the spin connection. Besides that, with the expressions (8), (9), (10), (11) and (12), we obtain

$$
\begin{aligned}
\Gamma_{t}(x) & =-\frac{1}{2} \frac{\kappa \Omega \eta}{\sqrt{1-\kappa^{2}}} \alpha^{1}-\frac{i}{2} \frac{\Omega \eta}{\sqrt{1-\kappa^{2}}} \Sigma^{3}, \\
\Gamma_{\rho}(x) & =\frac{1}{2} \frac{\Omega \eta}{\left(1-\kappa^{2}\right)} \alpha^{2}, \\
\Gamma_{\varphi}(x) & =-\frac{1}{2} \frac{\kappa \eta}{\sqrt{1-\kappa^{2}}} \alpha^{1}-\frac{i}{2} \frac{\eta}{\sqrt{1-\kappa^{2}}} \Sigma^{3},
\end{aligned}
$$

where implies

$\gamma^{\mu}(x) \Gamma_{\mu}(x)=\frac{1}{2} \frac{i \eta \gamma^{0} \Omega}{\left(1-\kappa^{2}\right)^{3 / 2}} \Sigma^{3}+\frac{1}{2 \rho} \gamma^{1}$.

With respect to curved gamma matrices, we have

$$
\begin{aligned}
& \gamma^{0}(x)=\frac{1}{\sqrt{1-\kappa^{2}}} \gamma^{0}+\frac{\kappa}{\sqrt{1-\kappa^{2}}} \gamma^{2}, \\
& \gamma^{1}(x)=\frac{1}{\sqrt{1-\kappa^{2}}} \gamma^{1}, \\
& \gamma^{2}(x)=\frac{\sqrt{1-\kappa^{2}}}{\eta \rho} \gamma^{2}, \\
& \gamma^{3}(x)=\gamma^{3} .
\end{aligned}
$$


Therefore, using the informations here presented and of the Sect. 2, Eq. (15) becomes

$$
\begin{aligned}
& \frac{i}{\sqrt{1-\kappa^{2}}}\left[\left(\gamma^{0}+\kappa \gamma^{2}\right) \partial_{t}+\gamma^{1}\left(\partial_{\rho}+m_{0} \omega \rho \gamma^{0}\right)\right] \Psi+\frac{i}{2 \rho} \gamma^{1} \Psi \\
& +\frac{i \gamma^{2}}{\eta \rho} \sqrt{1-\kappa^{2}} \partial_{\varphi} \Psi+i \gamma^{3} \partial_{z} \Psi-\frac{\eta \gamma^{0}}{\left(1-\kappa^{2}\right)^{3 / 2}} S \cdot \Omega \Psi \\
& +\frac{i \mu \lambda}{\eta \rho}\left[\frac{1}{\sqrt{1-\kappa^{2}}} \gamma^{0}+\frac{\kappa}{\sqrt{1-\kappa^{2}}} \gamma^{2}\right] \gamma^{1} \Psi-m_{0} \Psi=0,
\end{aligned}
$$

where the term $\boldsymbol{S} \cdot \boldsymbol{\Omega} S \cdot \Omega$ is so-called spin-rotation coupling [31,89], being $\boldsymbol{\Omega}=\Omega \hat{\mathbf{z}}$ and $\boldsymbol{S}=\frac{1}{2} \boldsymbol{\Sigma}$ is the spin operator [87].

We see that it is difficult to proceed without the simplification of Eq. (24). Thus, to solve exactly Eq. (24), we consider that the linear velocity of the rotation frame being small compared with the velocity of the light; consequently, we have $\kappa^{2} \ll 1[75,86]$. Using this condition and adopting the polar coordinates system where $\partial_{z} \Psi=0$, we get

$$
\begin{aligned}
& i \gamma^{0} \partial_{t} \Psi+i \gamma^{1}\left[\partial_{\rho}+\frac{1}{2 \rho}+\left(m_{0} \omega \rho-\frac{\mu \lambda}{\eta \rho}\right) \gamma^{0}\right] \Psi-m_{0} \Psi \\
& +i \gamma^{2}\left[\frac{1}{\eta \rho} \partial_{\varphi}+\Omega \eta \rho \partial_{t}\right] \Psi-\eta \gamma^{0} \boldsymbol{S} \cdot \boldsymbol{\Omega} S \cdot \Omega \Psi+i \mu \lambda \Omega \gamma^{2} \gamma^{1} \Psi .
\end{aligned}
$$

Since we are working planar spacetime, it is convenient to write the matrices $\gamma=\left(\gamma^{1}, \gamma^{2}, 0\right)=\left(-\gamma_{1},-\gamma_{2}, 0\right)$ and $\gamma^{0}$ and $\boldsymbol{S}=\left(0,0, \frac{1}{2} \Sigma^{3}\right)$ in terms of the Pauli matrices, i.e., $\gamma_{1}=\sigma_{3} \sigma_{1}=i \sigma_{2}, \gamma_{2}=s \sigma_{3} \sigma_{2}=-i s \sigma_{1}$ and $\gamma^{0}=\Sigma^{3}=$ $\sigma_{3}[71,78,79,87]$. By using this information and setting the following ansatz for the two-component Dirac spinor [71,88]

$\Psi(t, \rho, \varphi)=e^{i\left(m_{l} \varphi-E t\right)}\left(\begin{array}{c}R_{+}(\rho) \\ i R_{-}(\rho)\end{array}\right)$,

we have from (25) a system of two first-order coupled differential equations as

$$
\begin{aligned}
& \left(M_{0}-\mathscr{E}\right) R_{+}(\rho)=\left[\frac{d}{d \rho}-m_{0} \bar{\Omega} \rho+\frac{s}{\eta \rho} N_{+}\right] R_{-}(\rho), \\
& \left(M_{0}+\mathscr{E}\right) R_{-}(\rho)=\left[\frac{d}{d \rho}+m_{0} \bar{\Omega} \rho-\frac{s}{\eta \rho} N_{-}\right] R_{+}(\rho),
\end{aligned}
$$

where

$$
\begin{gathered}
M_{0} \equiv m_{0}+\frac{\eta \Omega}{2}, \quad \mathscr{E} \equiv E-\frac{\Phi_{A C} \Omega}{2 \pi}, \\
N_{ \pm} \equiv m_{l}+\frac{\Phi_{A C}}{2 \pi} \pm \frac{s \eta}{2},
\end{gathered}
$$

where $\bar{\Omega} \equiv\left(\omega+s \frac{\eta \Omega E}{m_{0}}\right)>0$ is an effective angular frequency, $E$ is the total relativistic energy, $m_{l}=$ $\pm 1 / 2, \pm 3 / 2, \ldots$ is the orbital magnetic quantum number and $\Phi_{A C}=2 \pi s \mu \lambda$ is a topological quantum phase, also so-called of AC quantum phase, and $s= \pm 1$ corresponds to projections of the MDM of the particle along on the $z$-axis (aligned or unaligned with the spin) [57,81].

Substituting now (28) into (27) and vice versa, we obtain two differential equations written compactly in the form

$$
\left[\frac{d^{2}}{d \rho^{2}}+\frac{1}{\rho} \frac{d}{d \rho}-\frac{\gamma_{r}^{2}}{\eta^{2} \rho^{2}}-(m \bar{\Omega} \rho)^{2}+E_{r}\right] R_{r}(\rho)=0,
$$

where

$$
\begin{aligned}
\gamma_{r} \equiv & m_{l}+\frac{\Phi}{\Phi_{0}}-\frac{r s \eta}{2}, \quad E_{r} \equiv \mathscr{E}^{2}-M_{0}^{2} \\
& +\frac{2 s m_{0} \bar{\Omega}}{\eta} \gamma_{r}+2 r m_{0} \bar{\Omega},
\end{aligned}
$$

being $R_{r}(\rho)$ real radial functions and the parameter $r$ characterizes the two components of the spinor, being that $r=+1$ describes a particle with $\operatorname{spin} u p(s=+1)$ or down $(s=-1)$ and $r=-1$ describes a antiparticle with spin up $(s=+1)$ or down $(s=-1)$, respectively.

In order to solve Eq. (30), we will introduce a new dimensionless variable given by $\tau=m_{0} \bar{\Omega} \rho^{2}$. Thereby, through a change of variable, Eq. (30) becomes

$$
\left[\tau \frac{d^{2}}{d \tau^{2}}+\frac{d}{d \tau}-\frac{\gamma_{r}^{2}}{4 \eta^{2} \tau}-\frac{\tau}{4}+\bar{E}_{r}\right] R_{r}(\tau)=0,
$$

where

$\bar{E}_{r} \equiv \frac{E_{r}}{4 m_{0} \bar{\Omega}}$.

Analyzing now the asymptotic behavior of Eq. (32) for $\tau \rightarrow 0$ and $\tau \rightarrow \infty$, we obtain a regular solution for this equation given by the following ansatz

$R_{r}(\tau)=\tau^{\frac{|\gamma r|}{2 \eta}} e^{-\frac{\tau}{2}} F_{r}(\tau)$

where $F_{r}(\tau)$ are unknown functions to be determined, and $R_{r}(\tau)$ must satisfy the following boundary conditions to be a physically acceptable solution (normalizable solution)

$R_{r}(\tau \rightarrow 0)=R_{r}(\tau \rightarrow \infty)=0$.

In this way, substituting (34) into Eq. (32), we obtain

$$
\left[\tau \frac{d^{2}}{d \tau^{2}}+\left(\left|\bar{\gamma}_{r}\right|-\tau\right) \frac{d}{d \tau}+\left(\bar{E}_{r}-\frac{\left|\bar{\gamma}_{r}\right|}{2}\right)\right] F_{r}(\tau)=0,
$$

where

$\left|\bar{\gamma}_{r}\right| \equiv \frac{\left|\gamma_{r}\right|}{\eta}+1$

It is not difficult to note that Eq. (36) is a the confluent hypergeometric equation, whose solution is the confluent hypergeometric function and is denoted by $[78,90]$

$F_{r}(\tau)={ }_{1} F_{1}\left(\frac{\left|\bar{\gamma}_{r}\right|}{2}-\bar{E}_{r},\left|\bar{\gamma}_{r}\right| ; \tau\right)$.

However, for that the Dirac spinor becomes a solution finite (normalizable), the confluent hypergeometric function 
must become a polynomial of degree $n$, consequently, the parameter $\frac{\left|\bar{\gamma}_{r}\right|}{2}-\bar{E}_{r}$ must to be equal to a non-positive integer number $-n(n=0,1,2, \ldots)$. Therefore, we obtain from this condition (quantization condition) the following relativistic energy spectrum of the $2 \mathrm{D} D O$ in the presence of the $\mathrm{AC}$ effect and under the influence of topological, noninertial and spin effects

$$
\begin{aligned}
& E_{n_{r}, m_{l}, s}^{\sigma}=\sigma \sqrt{\left(2 \eta \Omega N_{r}\right)^{2}+M_{0}^{2}+4 N_{r}\left(\frac{s \eta \Omega^{2} \Phi_{A C}}{2 \pi}+m_{0} \omega\right)} \\
& +\Omega\left(\frac{\Phi_{A C}}{2 \pi}+2 s \eta N_{r}\right),
\end{aligned}
$$

where

$N_{r} \equiv\left(n_{r}+\frac{\Gamma_{r}}{2 \eta}\right)$,

being $n_{r} \equiv n+\frac{1-r}{2}$ a quantum number, $\sigma=+1$ corresponds to the positive energy states (particle or DO), $\sigma=-1$ corresponds to the negative energy states (antiparticle or anti-DO) and the quantity $\Gamma_{r}$ is given by $\Gamma_{r} \equiv\left|\gamma_{r}\right|-s \gamma_{r}$. We see that the spectrum (39) explicitly depends on the spin parameter $s$, quantum phase $\Phi_{A C}$, angular velocity $\Omega$ of the rotating frame, and of the deficit angle $\eta$ generated by cosmic string. In particular, the rotating frame breaks the periodicity of the spectrum, since for $\Omega=0$ we have a spectrum with periodicity $\pm 2 \pi$ [81]. Although the uniformly charged wire which generates the electric field of the AC effect be located on the axis of symmetry of the cosmic string, a region forbidden, we see that the energies of the DO has a contribution due to conical singularity generated by the cosmic string. We note that for $r=s=+1$ the terms of the spectrum (39) are summed, while for $r=+1$ and $s=-1$, the terms are subtracted, consequently, the energies of the particle with spin up are larger than with spin down. On the other hand, we note that for $r=s=-1$ the terms of the spectrum (39) are summed, while for $r=-1$ and $s=+1$, the terms are subtracted, consequently, the energies (in absolute values) of the antiparticle with spin down are larger than with spin up. Moreover, we verified that the parameters $\eta, \Omega$ and $\Phi_{A C}$ have the function of increasing the energies of the spectrum, i.e., in the limits $\eta \rightarrow 0$ (extremely dense cosmic string, $\Omega \rightarrow \infty$ or $\Phi_{A C} \rightarrow \infty$, we have $\left|E_{n_{s}, m_{l}, s}^{\sigma}\right| \rightarrow \infty$.

Now, comparing the spectrum (39) with the literature, we verified that in the absence of the $\mathrm{AC} \operatorname{effect}\left(\Phi_{A C}=0\right)$ and of the topological and spin effects $(\eta=s=+1)$ with $m_{l}>0$, we obtain the spectrum of the DO in a rotating frame [86]. Already in the absence of the AC effect $\left(\Phi_{A C}=0\right)$ and of the topological and noninertial effects $(\eta=+1$ and $\Omega=0$ ), we obtain the spectrum of the DO in a flat inertial frame with and without the influence of the spin effects $[78,79,88]$. From the above, we see that the spectrum (39) generalizes some relativistic particular planar cases of the literature when $\Omega$, $\Phi_{A C}, s$, or $\eta$ are excluded of the system.
From here on let us concentrate on the form of the twocomponent Dirac spinor for the bound states of the system. Therefore, substituting the variable $\tau=m_{0} \bar{\Omega} \rho^{2}$ in the radial functions (34), we obtain the following Dirac spinor

$$
\Psi=e^{i\left(m_{l} \varphi-E t\right)}\left(\begin{array}{c}
C_{+} \rho^{\frac{|\gamma+|}{\eta}} e^{-\frac{\lambda \rho^{2}}{2}}{ }_{1} F_{1}\left(-n,\left|\bar{\gamma}_{+}\right| ; \lambda \rho^{2}\right) \\
i C_{-} \rho^{\frac{|\gamma-|}{\eta}} e^{-\frac{\lambda \rho^{2}}{2}}{ }_{1} F_{1}\left(-n,\left|\bar{\gamma}_{-}\right| ; \lambda \rho^{2}\right)
\end{array}\right),
$$

where

$$
C_{r} \equiv\left(m_{0} \bar{\Omega}\right)^{\frac{|\gamma r|}{2 \eta}}, \quad \lambda \equiv m_{0} \bar{\Omega}, \quad(r= \pm 1) .
$$

It should be noted that our Dirac spinor simultaneously incorporates the positive and negative values of the quantum number $m_{l}$, which does not happen, for instance, in Ref. [88]. A of the advantages of we have a spinor with this characteristic is the possibility of calculating the physical observables more faster and direct than if we had two spinors.

\section{Nonrelativistic limit}

In this section, we analyze the nonrelativistic limit of our results. To get this limit is necessary to consider that most of the total energy of the system stay concentrated in the rest energy of the particle [87], i.e., $E \cong \varepsilon+m_{0}$, where $m_{0} \gg \varepsilon$ and $m_{0} \gg \omega$. So, using this prescription in Eq. (30), we get

$$
\begin{aligned}
& {\left[H_{Q H O}-\frac{r \bar{\Omega}}{2}-\frac{2 \bar{\Omega}}{\eta} \mathbf{S} \cdot \mathbf{L}+r \eta \boldsymbol{S} \cdot \boldsymbol{\Omega} S \cdot \Omega-\frac{\Phi_{A C}}{2 \pi}\left(\Omega-r \frac{\bar{\Omega}}{\eta}\right)\right]_{\psi}} \\
& \quad=\varepsilon \psi,
\end{aligned}
$$

where

$H_{Q H O}=-\frac{1}{2 m_{0}}\left(\frac{\partial^{2}}{\partial \rho^{2}}+\frac{1}{\rho} \frac{\partial}{\partial \rho}-\frac{\ell_{z}^{2}}{\eta^{2} \rho^{2}}\right)+\frac{1}{2} m_{0} \bar{\Omega}^{2} \rho^{2}$,

with

$$
\begin{aligned}
\ell_{z} & =L_{z}+\frac{\Phi_{A C}}{2 \pi}-\frac{\eta}{2}, \bar{\Omega}=\omega+2 \eta S \cdot \Omega S \cdot \Omega, \quad \Omega=\Omega \hat{\mathbf{z}} \\
S & =\frac{1}{2} \sigma, \quad L_{z}=-i \frac{\partial}{\partial \varphi}
\end{aligned}
$$

being $\psi=e^{i m_{l} \varphi}\left(R_{+}(\rho), R_{-}(\rho)\right)^{T}$ the Pauli spinor and satisfies $\sigma_{3} \psi(\rho, \varphi)=r \psi(\rho, \varphi)$. Here, we have $r \equiv s= \pm 1$ and $r s=1$, where $r=+1$ describes a particle with up and $r=-1$ describes a particle with down, respectively. We verify that the first term in (43) represent the Hamiltonian of the 2D QHO in the presence of the AC effect and under the influence of topological, noninertial and spin effects. Here, the topological effects now are generated by a conic Euclidean space [91]. Besides, the third term in (43) describes a spinorbit coupling, represented by $\mathbf{S} \cdot \mathbf{L}$, and of strength $\bar{\Omega} / \hbar$ 
(restoring the factor $\hbar$ ), while the fourth term describes the spin-rotation coupling, represented by $\boldsymbol{S} \cdot \boldsymbol{\Omega}$, respectively.

Now, using the prescription $E \cong \varepsilon+m_{0}$ in (39), we obtain the following nonrelativistic energy spectrum of the $2 \mathrm{D} \mathrm{QHO}$ in the presence of the AC effect and under the influence of topological, noninertial and spin effects

$\varepsilon_{n_{r}, m_{l}}=\Omega\left(\frac{\Phi_{A C}}{2 \pi}+\frac{\eta}{2}\right)+2 N_{r}(r \eta \Omega+\omega)$.

We see that in addition of the spectrum (46) depend on the spin parameter $r$, AC quantum phase $\Phi_{A C}$, angular velocity $\Omega$ of the rotating frame, and of the deficit angle $\eta$ associated to topology of the conic space, grow infinitely in the limits $\eta \rightarrow 0, \Omega \rightarrow \infty$ or $\Phi_{A C} \rightarrow \infty$. We see that for $r=+1$ the terms of the spectrum (46) are summed, while for $r=-1$ , the terms are subtracted, i.e., the energies of the particle (or QHO) with spin up are larger than with spin down. Now, comparing the spectrum (46) with the literature, we verified that in the absence of the $\mathrm{AC}$ effect $\left(\Phi_{A C}=0\right)$ and of the topological and spin effects $(\eta=r=+1)$ with $m_{l}>0$, we obtain the spectrum of the QHO in a rotating frame [86]. Already in the absence of the $\operatorname{AC} \operatorname{effect}\left(\Phi_{A C}=0\right)$ and of the topological and noninertial effects $(\eta=+1$ and $\Omega=0)$, we obtain the spectrum of the OHQ in a flat inertial frame under the influence of the spin effects [78]. From the above, we see that the spectrum (46) generalizes some nonrelativistic particular planar cases of the literature when $\Omega, \Phi_{A C}, r$, or $\eta$ are excluded of the system.

\section{Conclusions}

In this paper, we study the influence of topological, spin and noninertial effects on the relativistic and nonrelativistic quantum dynamics of the 2D DO in the presence of the AC effect. Next, we determine the relativistic bound-state solutions of the system, given by two-component Dirac spinor and the energy spectrum.In particular, we verify that this spinor is written in terms of the confluent hypergeometric functions and this spectrum depends on the quantum numbers $n$ and $m_{l}$, parameters $s$ and $\eta$ associated to the topological and spin effects, quantum phase $\Phi_{A C}$, and of the angular velocity $\Omega$ of the rotating frame. We note that besides of the rotating frame break the periodicity of the spectrum, in the limits $\eta \rightarrow 0$ (extremely dense cosmic string), $\Omega \rightarrow \infty$ or $\Phi_{A C} \rightarrow \infty$ the spectrum grows infinitely. On the other hand, we note that the energies of the particle (or DO) with spin up are larger than with spin down. However, we also note that the energies of the antiparticle (or anti-DO) with spin down are larger than with spin up. Now, comparing our energy spectrum with other works, we verified that this spectrum generalizes some relativistic particular planar cases of the literature when $\Omega$, $\Phi_{A C}, s$ or $\eta$ are excluded of the system.
Finally, we study the nonrelativistic limit of our results. For instance, considering consider that most of the total energy of the system stay concentrated in the rest energy of the particle, we obtain the motion equation of the $2 \mathrm{D}$ $\mathrm{QHO}$ in the presence of the $\mathrm{AC}$ effect and under the influence of topological, spin and noninertial effects. We verified that these topological effects are generated by a conic space and the oscillator has two types of couplings: the spin-orbit coupling, given by $\mathbf{S} \cdot \mathbf{L}$, and the spin-rotation coupling, given by $\boldsymbol{S} \cdot \boldsymbol{\Omega}$. With respect to the nonrelativistic energy spectrum, such spectrum has some similarities with the relativistic case, for instance, depend of $n, m_{l}, s, \Phi_{A C}, \Omega$ and $\eta$, not is a periodic function and the parameters $\eta, \Omega$ and $\Phi_{A C}$ have the function of increasing the energies. Now, comparing our energy spectrum with other works, we verified that this spectrum generalizes some nonrelativistic particular planar cases of the literature when $\Omega, \Phi_{A C}, s$ or $\eta$ are excluded of the system.

Acknowledgements The author would like to thank the Conselho Nacional de Desenvolvimento Científico e Tecnológico (CNPq) for financial support.

Data Availability Statement This manuscript has no associated data or the data will not be deposited. [Author's comment: There is no data because this is theoretical work based on calculations to describe the relativistic and nonrelativistic quantum dynamics of Dirac oscillator.]

Open Access This article is distributed under the terms of the Creative Commons Attribution 4.0 International License (http://creativecomm ons.org/licenses/by/4.0/), which permits unrestricted use, distribution, and reproduction in any medium, provided you give appropriate credit to the original author(s) and the source, provide a link to the Creative Commons license, and indicate if changes were made. Funded by SCOAP $^{3}$.

\section{References}

1. M. Moshinsky, A. Szczepaniak, J. Phys. A: Math. Gen. 22, L817 (1989)

2. A. Boumali, H. Hassanabadi, Eur. Phys. J. Plus 128, 124 (2013)

3. H. Hassanabadi, S. Sargolzaeipor, B.H. Yazarloo, Few-Body Syst. 56, $115(2015)$

4. J. Munarriz, F. Dominguez-Adame, R.P.A. Lima, Phys. Lett. A 376, 3475 (2012)

5. D.A. Kulikov, I.V. Uvarov, A.P. Yaroshenko, Cent. Eur. J. Phys. 11, 1006 (2013). arXiv: 1112.4653 [hep-ph]

6. J. Grineviciute, D. Halderson, Phys. Rev. C 85, 054617 (2012). arXiv: 1404.4170 [nucl-th]

7. M. Moshinsky, G. Loyola, Found. Phys. 23, 197 (1993)

8. O.L. de Lange, J. Math. Phys. 32, 1296 (1991)

9. A. Bermudez, M.A. Martin-Delgado, E. Solano, Phys. Rev. A 76, 041801 (2007). arXiv:0704.2315 [quant-ph]

10. S. Longhi, Opt. Lett. 35, 1302 (2010). arXiv:1009.0159 [physics.optics]

11. C. Quimbay, P. Strange, Graphene physics via the Dirac oscillator in (2+1) dimensions (2013). arXiv:1311.2021 [cond-mat.mes-hall]

12. A. Boumali, Phys. Scrip. 90, 045702 (2015). arXiv:1411.1353 [cond-mat.mes-hall] 
13. A. Belouad, A. Jellal, Y. Zahidi, Phys. Lett. A 380, 773 (2016). arXiv:1505.08068 [cond-mat.mes-hall]

14. A. Bermudez, M. Martin-Delgado, A. Luis, Phys. Rev. A 77, 063815 (2008). arXiv:0802.0577 [quant-ph]

15. C. Quimbay, P. Strange, Quantum phase transition in the chirality of the (2+1)-dimensional Dirac oscillator (2013). arXiv:1312.5251 [hep-th]

16. B.P. Mandal, S.K. Rai, Phys. Lett. A 376, 2467 (2012). arXiv: 1203.2714 [hep-th]

17. G. Melo, M. Montigny, P. Pompeia, E. Santos, Int. J. Theor. Phys. 52, $441(2013)$

18. C. Quesne, V.M. Tkachuk, J. Phys. A: Math. Gen. 38, 1747 (2005). arXiv:math-ph/0412052

19. L. Menculini, O. Panella, P. Roy, Phys. Rev. D 91, 045032 (2015). arXiv:1411.5278 [quant-ph]

20. Int Quesne, J. Mod. Phys. A 6, 1567 (1991)

21. G. Junker, A. Inomata, J. Math. Phys. 59, 052301 (2018). arXiv:1712.08759 [quant-ph]

22. J.A. Franco-Villafañe, E. Sadurní, S. Barkhofen, U. Kuhl, F. Mortessagne, T.H. Seligman, Phys. Rev. Lett. 111, 170405 (2013). arXiv:1306.2204 [cond-mat.mes-hall]

23. C.L. Ho, P. Roy, Europhys. Lett. 124, 60003 (2019). arXiv:1808.03962 [quant-ph]

24. R.R.S. Oliveira, R.V. Maluf, C.A.S. Almeida, Ann. Phys. 400, 1 (2019). arXiv:1809.03801 [quant-ph]

25. M. Salazar-Ramírez, D. Ojeda-Guillén, A. Morales-González, V.H. García-Ortega, Eur. Phys. J. Plus 134, 8 (2019). arXiv:1806.05329 [math-ph]

26. M. Hosseinpour, H. Hassanabadi, M. de Montigny, Eur. Phys. J. C 79, 311 (2019). arXiv:1904.05889 [hep-th]

27. M. Matsuo, J.I. Ieda, E. Saitoh, S. Maekawa, Phys. Rev. B 84, 104410 (2011). arXiv:1106.0366 [cond-mat.mes-hall]

28. M.G. Sagnac, C.R. Acad, Sci. (Paris) 157, 708 (1913)

29. S.J. Barnett, Phys. Rev. 6, 239 (1915)

30. A. Einstein, W.J. de Haas, Verh. Dtsch. Phys. Ges. 17, 152 (1915)

31. B. Mashhoon, Phys. Rev. Lett. 61, 2639 (1988)

32. U.R. Fischer, N. Schopohl, Europhys. Lett. 54, 502 (2001). arXiv:cond-mat/0004339 [cond-mat.mes-hall]

33. S. Viefers, J. Phys, Condens. Matter 20, 123202 (2008). arXiv:0801.4856 [cond-mat.mes-hall]

34. V. Schweikhard, I. Coddington, P. Engels, V.P. Mogendorff, E.A. Cornell, Phys. Rev. Lett. 92, 040404 (2004). arXiv:cond-mat/0308582 [cond-mat.mes-hall]

35. N.R. Cooper, N.K. Wilkin, J.M.F. Gunn, Phys. Rev. Lett. 87, 120405 (2001). arXiv:cond-mat/0107005 [cond- mat]

36. J.Q. Shen, S.L. He, Phys. Rev. B 68, 195421 (2003). arXiv:quant-ph/0301094 [quant-ph]

37. J.R. Lima, J. Brandão, M.M. Cunha, F. Moraes, Eur. Phys. J. D 68, 94 (2014). arXiv:1405.6633 [cond-mat.mes-hall]

38. N.R. Cooper, Adv. Phys. 57, 539 (2008). arXiv:0810.4398 [condmat.mes-hall]

39. L.H. Lu, Y.Q. Li, Phys. Rev. A 76, 023410 (2007). arXiv:cond-mat/0701481 [cond-mat.other]

40. B.Q. Wang, Z.W. Long, C.Y. Long, S.R. Wu, Mod. Phys. Lett. A 33, 1850025 (2018)

41. K. Konno, R. Takahashi, Phys. Rev. D 85, 061502 (2012). arXiv:1201.5188v1 [gr-qc]

42. L.C.N. Santos, C.C. Barros, Eur. Phys. J. C 78, 13 (2018). arXiv:1801.01024 [hep-th]

43. M. Hosseinpour, H. Hassanabadi, Eur. Phys. J. Plus 130, 236 (2015). arXiv:1505.00096 [hep-th]

44. L.B. Castro, Eur. Phys. J. C 76, 61 (2016). arXiv:1511.01939 [hepth]

45. Ö.F. Dayi, E. Yunt, Ann. Phys. 390, 143 (2018). arXiv:1705.07590v1 [math-ph]
46. M. Matsuo, J.I. Ieda, E. Saitoh, S. Maekawa, Phys. Rev. Lett. 106, 076601 (2011). arXiv:1009.5424 [cond-mat.mes-hall]

47. J. Anandan, Phys. Rev. D 24, 338 (1981)

48. M.A. Zubkov, Europhys. Lett. 121, 47001 (2018). arXiv:1801.05368 [hep-ph]

49. M.N. Chernodub, S. Gongyo, J. High Energy Phys. 2017, 136 (2017). arXiv:1611.02598 [hep-th]

50. Y. Liu, I. Zahed, Phys. Rev. D 98, 014017 (2018). arXiv:1710.02895 [hep-ph]

51. M.N. Chernodub, S. Gongyo, Phys. Rev. D 96, 096014 (2017). arXiv: 1706.08448 [hep-th]

52. E. Cavalcante, J. Carvalho, C. Furtado, Eur. Phys. J. Plus 131, 288 (2016)

53. J. Gonzalez, F. Guinea, M.A.H. Vozmediano, Nucl. Phys. B 406, 771 (1993)

54. D.V. Kolesnikov, V.A. Osipov, Eur. Phys. J. B 49, 465 (2006). arXiv:cond-mat/0510636 [cond-mat.mtrl-sci]

55. M.M. Cunha, J. Brandão, J.R. Lima, F. Moraes, Eur. Phys. J. B 88, $288(2015)$

56. F.A. Gomes, V.B. Bezerra, J.R.F. de Lima, F.J.S. Moraes, Eur. Phys. J. B 92, 41 (2019). arXiv:1806.01324 [cond-mat.mes-hall]

57. Y. Aharonov, A. Casher, Phys. Rev. Lett. 53, 319 (1984)

58. A. Cimmino, G.I. Opat, A.G. Klein, H. Kaiser, S.A. Werner, M. Arif, R. Clothier, Phys. Rev. Lett. 63, 380 (1989)

59. K. Sangster, E.A. Hinds, S.M. Barnett, E. Riis, Phys. Rev. Lett. 71, 3641 (1993)

60. C.R. Hagen, Phys. Rev. Lett. 64, 2347 (1990)

61. S. Bruce, L. Roa, C. Saavedra, A.B. Klimov, Phys Rev. A 60, R1 (1999). arXiv:quant-ph/9905074

62. B. Mirza, M. Zarei, Eur. Phys. J. C 32, 583 (2004). arXiv:hep-th/0311222

63. N.M.R. Peres, E.V. Castro, J. Phys. Condens. Matter 19, 406231 (2007). arXiv:0709.0646 [cond-mat.mes-hall]

64. Z.Z. Alisultanov, M.S. Reis, Europhys. Lett. 113, 28004 (2016). arXiv: 1510.06687 [cond-mat.mes-hall]

65. A.I. Nikishov, Sov. Phys. JETP 30, 660 (1970)

66. S.P. Kim, D.N. Page, Phys. Rev. D 75, 045013 (2007)

67. N. Chair, M.M. Sheikh-Jabbari, Phys. Lett. B 504, 141 (2001)

68. S.P. Gavrilov, D.M. Gitman, Phys. Rev. D 53, 7162 (1996)

69. E.R.B. de Mello, J. High Energy Phys. 2004, 016 (2004)

70. R. R. S. Oliveira, R. V. Maluf and C. A. S. Almeida, Exact solutions of the Dirac oscillator under the influence of the Aharonov-Casher effect in the cosmic string background (2018). arXiv:1810.11149 [quant-ph]

71. R.R.S. Oliveira, M.F. Sousa, Braz. J. Phys. 49, 315 (2019)

72. A.Y. Silenko, Russ. Phys. J. 48, 788 (2005)

73. K. Bakke, Open Phys. 10, 1089 (2012)

74. R.P. Martínez-y Romero, H.N. Núnez-Yépez, A.L. Salas-Brito, Eur. J. Phys. 16, 135 (1995)

75. K. Bakke, Eur. Phys. J. Plus 127, 82 (2012)

76. K. Bakke, Gen. Rel. Gravit. 45, 1847 (2013). arXiv:1307.2847 [quant-ph]

77. A. Vilenkin, E.P.S. Shellard, Cosmic Strings and Other Topological Defects (Cambridge University Press, Cambridge, 2000)

78. F.M. Andrade, E.O. Silva, Europhys. Lett. 108, 30003 (2014). arXiv: 1406.3249 [hep-th]

79. F.M. Andrade, E.O. Silva, Eur. Phys. J. C 74, 3187 (2014). arXiv:1403.4113 [hep-th]

80. J. Carvalho, C. Furtado, F. Moraes, Phys. Rev. A 84, 032109 (2011)

81. K. Bakke, C. Furtado, Ann. Phys. 336, 489 (2013)

82. M. Rojas, C. Filgueiras, J. Brandão, F. Moraes, Phys. Lett. A 382, 432 (2018). arXiv:1706.06421v1 [cond-mat.other]

83. M.O. Katanaev, I.V. Volovich, Ann. Phys. 216, 1 (1992)

84. H. Kleinert, Gauge Fields in Condensed Matter, vol. 2 (World Scientific, Singapore, 1989)

85. K. Bakke, C. Furtado, Phys. Rev. D 82, 084025 (2010) 
86. P. Strange, L.H. Ryder, Phys. Lett. A 380, 3465 (2016)

87. W. Greiner, Relativistic Quantum Mechanics: Wave Equations, vol. 3 (Springer, Berlin, 2000)

88. V.M. Villalba, Phys. Rev. A 49, 586 (1994)

89. F.W. Hehl, W.T. Ni, Phys. Rev. D 42, 2045 (1990)
90. M. Abramowitz, I.A. Stegum, Handbook of Mathematical Functions (Dover Publications Inc., New York, 1965)

91. F.M. Andrade, E.O. Silva, M. Pereira, Phys. Rev. D 85, 041701 (2012). arXiv:1112.0265 [quant-ph] 